\title{
Stable Disease
}

National Cancer Institute

\section{Source}

National Cancer Institute. Stable Disease. NCI Thesaurus. Code C18213.

Cancer that is neither decreasing nor increasing in extent or severity. 